\title{
EUV pattern defect detection sensitivity based on aerial image linewidth measurements
}

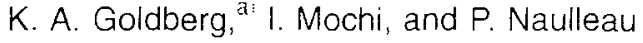 \\ Mail Stop 2-400, Lawrence Berkeley National Laboratory, Berkeley, California 94720 \\ T. Liang and P.-Y. Yan \\ Intel Corp., 3065 Bowers Avenue, Santa Clara, California 95054 \\ S. Huh \\ SEMATECH. 255 Fuller Road, Suite 309. Albany, New York 12203
}

(Received 7 July 2009; accepted 26 October 2009; published 3 December 2009)

\begin{abstract}
As the quality of EUV-wavelength mask inspection microscopes improves over time, the image properties and intensity profiles of reflected light can be evaluated in ever-greater detail. The SEMATECH Berkeley Actinic Inspection Tool (AIT) is one such microscope, featuring mask resolution values that match or exceed those available through lithographic printing in current photoresists. In order to evaluate the defect detection sensitivity of the AIT for dense line patterns on typical masks, the authors study the linewidth roughness (LWR) on two masks, as measured in the EUV images. They report the through-focus and pitch dependence of contrast, image log slope, linewidth, and LWR. The AIT currently reaches LWR $3 \sigma$ values close to $9 \mathrm{~nm}$ for $175 \mathrm{~nm}$ half-pitch lines. This value is below $10 \%$ linewidth for nearly all lines routinely measured in the AIT. Evidence suggests that this lower level may arise from the mask's inherent pattern roughness. While the sensitivity limit of the AIT has not yet been established, it is clear that the AIT has the required sensitivity to detect defects that cause 10\% linewidth changes in line sizes of $125 \mathrm{~nm}$ and larger. (C) 2009 American Vacuum Society. [DOI: 10.1116/1.3264676]
\end{abstract}

\section{INTRODUCTION}

Mask pattern inspection is essential to the development of every projection lithography technology. With shrinking design rules and complex optical proximity correction, the challenge grows with each technology generation. This is especially true for EUV lithography, in part because the development of extreme ultraviolet (EUV) wavelength microscopy is so new.

EUV-wavelength mask inspection microscopy has recently become available on prototype mask inspection systems. One such microscope is the SEMATECH Berkeley Actinic Inspection Tool (AIT), operating at Lawrence Berkeley National Laboratory. The AIT is a synchrotron-based Fresnel zoneplate microscope that provides high quality aerial image measurements of EUV reticles. ${ }^{1-4}$

Recent improvements in the AIT's resolution, ${ }^{2}$ imaging performance, ${ }^{+}$and illumination uniformity ${ }^{2}$ enable the images to be used for detailed line-intensity profile analysis. Such investigations include line properties such contrast, image slope, linewidth, and linewidth roughness (LWR). Typical experiments conducted with the AIT now include the measurement of patterned defects ${ }^{5}$ and the evaluation of defect repair strategies. ${ }^{6}$ Since the outcomes of these tests include the determination of whether or not given mask features qualify as printable defects, it is essential that we understand the measurement sensitivity of the microscope itself.

While many factors contribute to the observed noise and intensity fluctuations in the output images, the most relevant line property for this sensitivity assessment is the measured LWR. To measure defects with confidence, we must be able to separate real linewidth variations from random fluctuations. One commonly applied metric is the LWR $3 \sigma$ value. In the presence of random linewidth variations, with an assumed normal width distribution, the $3 \sigma$ value provides $99.7 \%$ confidence that an observed linewidth change greater than this amount is a true defect.

We report the image line-profile properties of two EUV masks that typify the recent measurements performed in the AIT. Mask 1 contains a buried array of bump-type substrate defects, below the multilayer-mirror coating, and an absorber pattern of large straight lines. This mask has been used in the measurement of defect sensitivity and for comparison with modeling. ${ }^{7.8}$ Our interest here is limited to the observed LWR properties, from which we can establish the AIT's measurement sensitivity to small linewidth changes. Mask 2 contains a pattern of dense straight lines (1:1 line to space ratio), with half-pitch values of $100 \mathrm{~nm}$ and higher. Mask 2 is a typical mask whose imaging properties are being evaluated in comparison with other masks. There are no intentional defects in the pattern areas we examined.

Knowing that the measured LWR comes from a combination of real effects and system noise, our measurements show that the AIT can detect mask pattern roughness for mask linewidths at or above $150 \mathrm{~nm}$. 


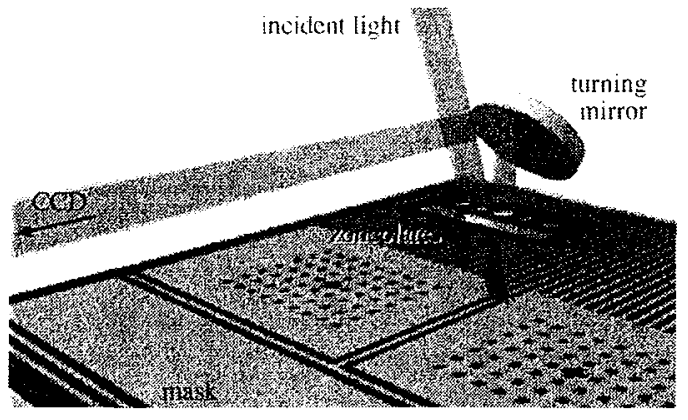

FIC. 1. AIT imaging optics and light path, not to scale. A zone plate array, held $750 \mu \mathrm{m}$ above the mask, projects a high-magnification image of the illuminated mask surface onto a CCD camera, after reflection form a $45^{\circ}$ multilayer-coated turning mirror

\section{SYSTEM DESCRIPTION}

Detailed descriptions of the AIT optical system and performance specifications have been presented previously. ${ }^{1.2}$ A schematic representation of the AIT's imaging optics is shown in Fig. 1. The AIT is a Fresnel zoneplate microscope that projects a highly magnified image of the light reflected from a mask's surface onto an EUV charge-coupled device (CCD) camera. A closely spaced array of zoneplates enables the user to select from objective lenses with different optical properties. In this way, the AIT can emulate the spatial resolution of various existing and future EUV lithography tools. For example, with $0.0625,0.0750$, and $0.0875 \mathrm{NA}$ objectives, the AIT emulates $4 \times$ demagnification projection tools with $0.25,0.30$, or $0.35 \mathrm{NA}$, beyond the current state of the art.

For the zone plates now installed in the AIT, the magnification ratio is $907 \times$. The $13.5 \mu \mathrm{m}$ square pixels on the CCD camera correspond to a $15 \mathrm{~nm}$ square area of the mask surface. This pixel density is several times higher than the resolution of the AIT, to allow appropriate sampling of the image.

\section{A. AlT illumination}

The AIT uses a synchrotron bending-magnet beamline at the Advanced Light Source, at Lawrence Berkeley National Laboratory (LBNL). A monochromator provides EUV illumination with a tunable wavelength and bandwidth: the AIT typically operates at $13.4 \mathrm{~nm}$ wavelength with a bandwidth, $\lambda / \Delta \lambda$, of 1500 . The narrow bandwidth is required to avoid chromatic aberration from the zoneplate lens.

Glancing-incidence beamline mirrors, including a Kirkpatrick-Baez mirror pair, create an intermediate focus of approximately $70 \times 300 \mu \mathrm{m}$, which is reimaged onto the mask by a $20 \times$ demagnification, EUV Schwarzschild objective. Using an off-axis subaperture of the Schwarzschild objective, the central ray of the illuminating beam is inclined at $6^{\circ}$ from vertical. Although the illumination solid half-angle is close to 0.0625 radians, the beamline's demagnification does not significantly break the coherence; we have experimen- tally measured partial coherence $\sigma$ values in the range of $0.1-0.2$, depending on the numerical aperture (NA) of the objective lens. ${ }^{.}$

\section{B. Wavelength-tuning through-focus measurements}

Because the zone plate lens focuses light by diffraction, its properties are wavelength sensitive. Tuning the illumination wavelength over a small range near $13.4 \mathrm{~nm}$ changes the zoneplate's focal length by up to several microns and enables a motion-free through-focus imaging capability with greater stability and reproducibility than can be achieved with mechanical stage motions. 'Beginning in October 2008, this has become the default through-focus-series data collection mode for the AIT.

\section{Zone plate optical alignment}

With $907 \times$ magnification, and a 1 in. ${ }^{2}$ CCD camera area, the AIT's viewable mask area is limited to a $30 \mu \mathrm{m}$-wide square in a single image. Ray tracing analysis shows that within that region, the aberrations (primarily astigmatism and coma) vary quickly away from the center point; ${ }^{4}$ the usable sweet spot of the lens, where the imaging performance may be considered diffraction limited, is only 5-8 $\mu \mathrm{m}$ diameter for most applications. With small zone plate position changes affecting the sweet spot's location, the persistent challenge for the AIT is maintaining knowledge of the field-dependent aberrations, so that the zone plate position can be optimized at the center of the field.

We have developed several tests, using fields of contacts, and other mask patterns, ${ }^{10}$ to quantitatively estimate the aberration magnitude at each point in the field. We rely on these tests, and other qualitative measures to maintain high data quality. The use of these aberration-measuring tests enables the AIT to achieve diffraction-limited wavefront quality. ${ }^{4,8}$

\section{Mask stage}

The AIT was originally designed with an unusual mask stage that now complicates imaging data analysis. The stage translates the mask in only one direction and rotates it in plane, under a stationary illuminating beam, to reach the measurement region. As a result, the orientation of measured line patterns seldom coincides with the conventional $x y$ axis defined by the CCD array. Image rotation is required to perform the types of analysis relevant to this work.

\section{IMAGE-SLOPE DEPENDENCE OF LWR}

Many of the factors that determine linewidth (LW) and LWR in printed images correspond directly to similar effects in the measurement of continuous-tone intensity images recorded by a mask inspection microscope. In photoresist, a printed line's width depends on the resist sensitivity, the exposure dose, and the shape of the line's intensity profile, in addition to other factors related to the resist processing. A simple way to evaluate linewidths in microscope images is to 


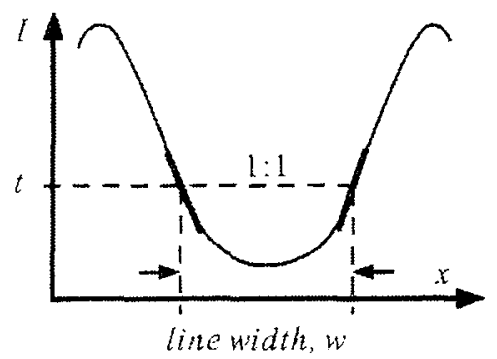

FIG. 2. Analysis of a single dark-line profile. A threshold intensity level $t$ is defined to achieve a dark linewidth equal to the space width. The absolute value of the intensity slope, measured at the threshold intensity, can be calculated from the average of the two sides.

apply a constant-intensity threshold to create a binary version of the image. This step approximates an ideal, homogeneous resist response.

Since the intensity slope at the edges of a printed line is never infinite, the LW will change with variations in the threshold value or with a global change in the intensity. In general, dark lines shrink when the global exposure level increases and expand when it falls. The rate of change depends directly on the intensity slope at the threshold level.

This approach is frequently cited in the derivation of the exposure latitude (EL), ${ }^{11.12}$ wherein the relationship between exposure dose change and LW change is calculated. Figure 2 illustrates the relationship between the intensity profile, the exposure threshold, and the LW.

Approximating the intensity profile as symmetric on the two sides of the line, we derive the following geometrically from Fig. 2:

$$
\Delta \mathrm{LW}=2 \frac{\partial x}{\partial I} \Delta I
$$

The factor of 2 accounts for width change contributions from both sides of the line. Here it is useful to introduce the image log slope (ILS) and the normalized image log slope (NILS) parameters," defined as follows:

$$
\begin{aligned}
& \mathrm{LS} \equiv \frac{I}{I} \frac{d I}{d x}=\frac{d \ln I}{d x}, \\
& \mathrm{NILS} \equiv \mathrm{LW} \frac{1}{I} \frac{d I}{d x}=\mathrm{LW} \frac{d \ln I}{d x} .
\end{aligned}
$$

By convention, these values are calculated at the intensity level that produces equal lines and spaces. ILS units are inverse length, while NILS is dimensionless. Noting that the global exposure level $E$ scales the local intensity profile $I$, we reach the conventional derivation of EL, ${ }^{12}$ here defined as $\Delta E / E$. From Eqs. (1) and (3) we have

$$
\frac{\Delta \mathrm{LW}}{\mathrm{LW}}=\frac{2}{\mathrm{NILS}} \frac{\Delta E}{E} .
$$

Statistically, the root-mean-square (rms) variation in measured LW (i.e., LWR), denoted as $\sigma_{\mathrm{LW}}$, will follow the rms variation in $E, \sigma_{E}$,

$$
\frac{\sigma_{L W}}{\text { LW }}=\frac{2}{\text { VILS }} \frac{\sigma_{E}}{E}
$$

However, in cases where the illuminating or the reflected intensity fluctuates on a scale comparable to or smaller than the lines being measured, a separate relationship emerges. When fine-scale point-to-point fluctuations are uncorrelated across the width of a single line, the line edge positions vary independently, and the dependence of $\sigma_{L W}$ on $\sigma_{E}$ is reduced by approximately $30 \%$.

$$
\frac{\sigma_{\mathrm{LW}}}{\mathrm{LW}}=\frac{\sqrt{2}}{\mathrm{NILS}} \frac{\sigma_{E}}{E} \text {. }
$$

The dependence of measured LW on spatially large or small intensity variations creates a NILS-dependent uniformity constraint for precise LW measurement in any mask pattern inspection tool, including the AIT. This constraint can be loosened in cases where the measured intensity can be reliably, mathematically normalized, and in cases where intensity variations across the measurement region are well known. The uniformity constraint thus applies to the postnormalization intensity and must be evaluated on a case-by-case basis.

Multilayer phase roughness, ${ }^{14}$ which creates an observable speckle pattern in mask images and printed lines, contributes local intensity variations that couple into the observed LWR in the manner described above. Depending on the relative size of the observed lines and the speckle pattern (which is affected by the NA, the illumination degree of partial coherence, and the lens' wavefront aberrations), Eqs. (5) and (6) become limiting cases used to describe the relationship between intensity variations and LW changes. In practice, the appropriate constant multiplier may be between these two values.

\section{MEASURING LWR AND OTHER LINE PROPERTIES IN IMAGE DATA}

Various systematic and random effects contribute to the LWR measured in mask images. Actual defects, inherent pattern roughness, and multilayer phase roughness all contribute to the line shapes we are trying to measure. These physical patterns are spatially filtered by the properties of the objective lens (finite NA, wavefront aberrations, etc.) and are affected by the illumination partial coherence. Scattered light, imperfections, or other diffraction orders in the zoneplate lens also contribute to local illumination-intensity variations that affect LW. In addition to these effects, CCD camera noise and dark current, and photon shot noise contribute random variations that increase the measured LWR. CCD images may also contain periodic noise patterns that are not entirely random in nature. Small distortions introduced by imperfections in the turning mirror between the zone plate and the CCD may also introduce small, stationary distortions. Furthermore, mechanical instability during the exposure can blur images in ways that can be difficult to measure accurately, especially when the instability is intermittant. 
The relative magnitudes of these nonilluminationdependent error sources are of great interest and are subjects of ongoing research. From the line-property data presented here and from the self-consistency of the results within individual measurement series, we conclude that these error sources are not yet limiting factors in line measurement with the AIT, for current linewidths of interest. Occasional mechanical instabilities are observed during measurement. and roughly 1 in 20 images is repeated to replace bad data.

In these studies, line properties are calculated from image-detail subregions that are 4 LW (2 cycles) wide by $2 \mu \mathrm{m}$ long. The $2 \mu \mathrm{m}$ length was chosen to be large enough to include a sufficient number of data points, yet small enough to minimize the effects of illumination nonuniformities (although a linear uniformity correction is applied).

The mask stage in the AIT translates in only one direction and rotates the mask in plane to reach the measurement re gion. For this reason, data analysis requires an imagerotation step, which is carefully preformed using bilinear interpolation onto a $5 \mathrm{~nm}$ pixel grid.

The line-intensity profiles used in the measurement of LW, contrast, NILS, and ILS are calculated by integrating the intensity along the $(2 \mu \mathrm{m})$ lines to form an average line profile. This step effectively utilizes 133 times $(2 \mu \mathrm{m}$ length $/ 15 \mathrm{~nm}$ per pixel) more image area than a single $15 \mathrm{~nm}$ wide row of the image detail extracted from the original measurement. Line averaging improves the signal-to-noise ratio (SNR), reducing the influence of random noise sources.

Measurements of LWR cannot take advantage of line averaging in the same way. After applying a threshold intensity level, an array of linewidth values is calculated, one from each row of the image detail. The LW is the average value, and the LWR is the standard deviation. (Typically, the LWR $3 \sigma$ value is reported.) LWR measurements rely on far less light per data point and are therefore much more sensitive to noise sources and the overall exposure level.

To emulate the behavior of photoresist-based experiments, it is important to select a self-consistent intensity threshold within each measurement series. The threshold intensity is used to determine LW, LWR, ILS, and NILS. Within each through-focus series, the image with peak contrast is identified as best focus. Within that image detail, we calculate the threshold level that provides equal lines and spaces (i.e., a 1:1 line-to-space ratio) and apply that level consistently to each image. This allows us to observe how LW, LWR, NILS, and ILS vary through focus. Separately, for Bossung calculations, ${ }^{15}$ we evaluate the LW at different intensitynormalized threshold levels, in uniform steps between 0 and 1.

\section{LWR MEASUREMENTS ON TWO TYPICAL MASKS}

The LWR and other image properties have been calculated for two masks typical of those inspected in the AIT in 2008-2009. Concentrating exclusively on pattern regions that do not contain intentional test defects reveals the AIT's

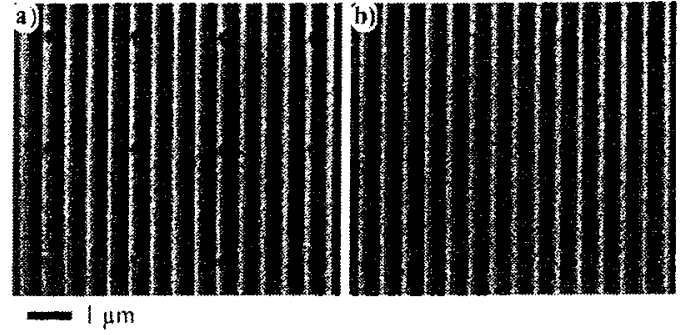

F!G. 3. Image details from mask 1 , with a $250 \mathrm{~nm}$ hp absorber pattern of dense lines above an array of buried bump-type phase defects. In the array region where the detects are large (a) their effect on the lines is clear. In a different part of the array (b), the line perturbations caused by the defects are close to or below the detection limit. LWR measurements in this article are based on line regions that exclude the known defect positions.

typical LWR value and allows us to calculate the pattern defect detection sensitivity limits of the AIT in real-world applications.

\section{A. Experiment conditions}

The zone plate used in all of these measurements has a mask-side NA of 0.0875 : equivalent to a $0.35 \mathrm{NA}, 4 \times$ stepper; higher than any of the EUV projection lithography prototype tools now in use. A $45 \mathrm{~s}$ exposure time was used for the mask 1 measurements. We increased the exposure time to $90 \mathrm{~s}$ for mask 2 to improve the SNR.

Through-focus data collection was performed with the wavelength-tuning technique (described in Ref. 3), using stationary mask and zone plate stages. Typically, between 13 and 17 steps through focus are collected, with an equivalent longitudinal mask-step size as small as $0.4 \mu \mathrm{m}$ for the smallest feature sizes. This step size is equivalent to $25 \mathrm{~nm}$ water step size in a $4 \times$ projection system.

\section{B. Mask 1}

The first mask under consideration has an absorber pattern of equal lines and spaces with $250 \mathrm{~nm}$ half-pitch, which is a relatively large feature size compared to other AIT measurements. Below the multilayer-mirror coating is a pattern of buried, bump-type, substrate defects measuring from 20 to $100 \mathrm{~nm}$ in height, which deform the top surface by up to $50 \mathrm{~nm}$ height, and $60 \mathrm{~nm}$ full width at half maximum. Images of these mask defects have been the subject of analysis and modeling." Figure 3 shows two regions of the defect array. In Fig. 3(a), the effect of the largest buried bumps are readily apparent; while in Fig. 3ib), the linewidth changes caused by much smaller defects [located in similar positions as those in Fig. 3(a)] are close to or below the detection limit. Figure 4 shows the measured ILS and NILS and LWR through-focus. Defect sizes are commonly defined relative to the linewidth; the vertical axes of Fig. 4 (b) show both physical size and relative size. 

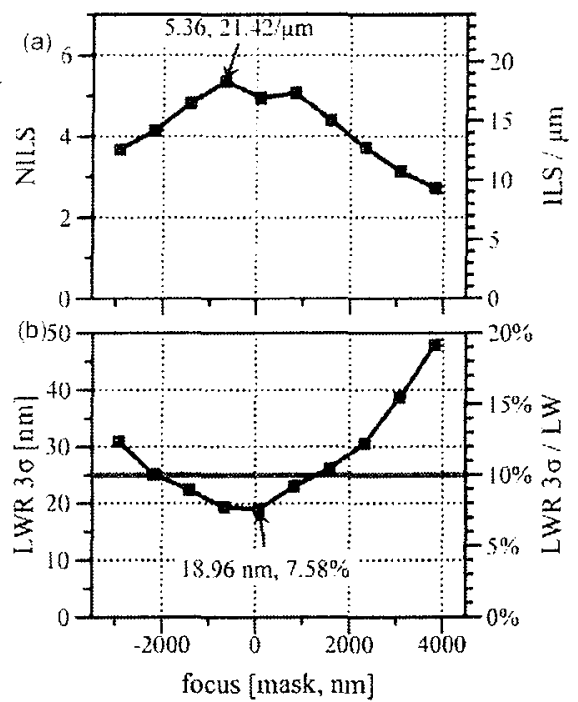

Fic. 4. Mask 1 line properties through focus. (a) NILS and ILS $\mu$ m. (b) LWR $3 \sigma$ and LWR $3 \sigma^{\prime} \mathrm{LW}$.

\section{Mask 2}

On the second mask, we inspected an array of dense-line features with multiple half-pitch values: 100, 125, 150, 175, and $200 \mathrm{~nm}$. Image details from various line sizes on mask 2 are shown in Fig. 5. Within the AIT's image area, the locations of the analyzed detail regions were selected based on the highest observed local contrast.

Because the image slope parameters (ILS and NILS) in Figs. 6ib) and 6(c) show a significant increase with LW, we anticipated a reduced sensitivity to noise in the LW and LWR measurements and a corresponding decrease in their values at best focus. However, for LWs of $150 \mathrm{~nm}$ and larger, the measured through-focus LWR $3 \sigma$ curves in Fig. 6(d) are nearly flat, with a lower limit just below $9 \mathrm{~nm}$. Although the absorber pattern LWR values for this mask have not been measured, this magnitude is consistent with the LWR $3 \sigma$ level observed from pattern roughness on other masks measured at LBNL." It is therefore likely that the observed lower limit arises from intrinsic mask properties, namely, pattern roughness.

The contribution of multilayer phase roughness to the LWR should also be considered. Measurements from bright areas between the line patterns show a speckle intensity

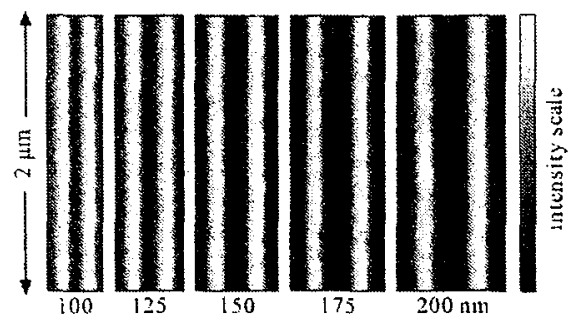

F:G. 5. Image details from dense line patterns on mask 2. The half-pitch values are shown below each detail. Detail regions measuring $2 \mu \mathrm{m} \times 4 \mathrm{LW}$, for each size, are used in the analysis of line properties through focus.
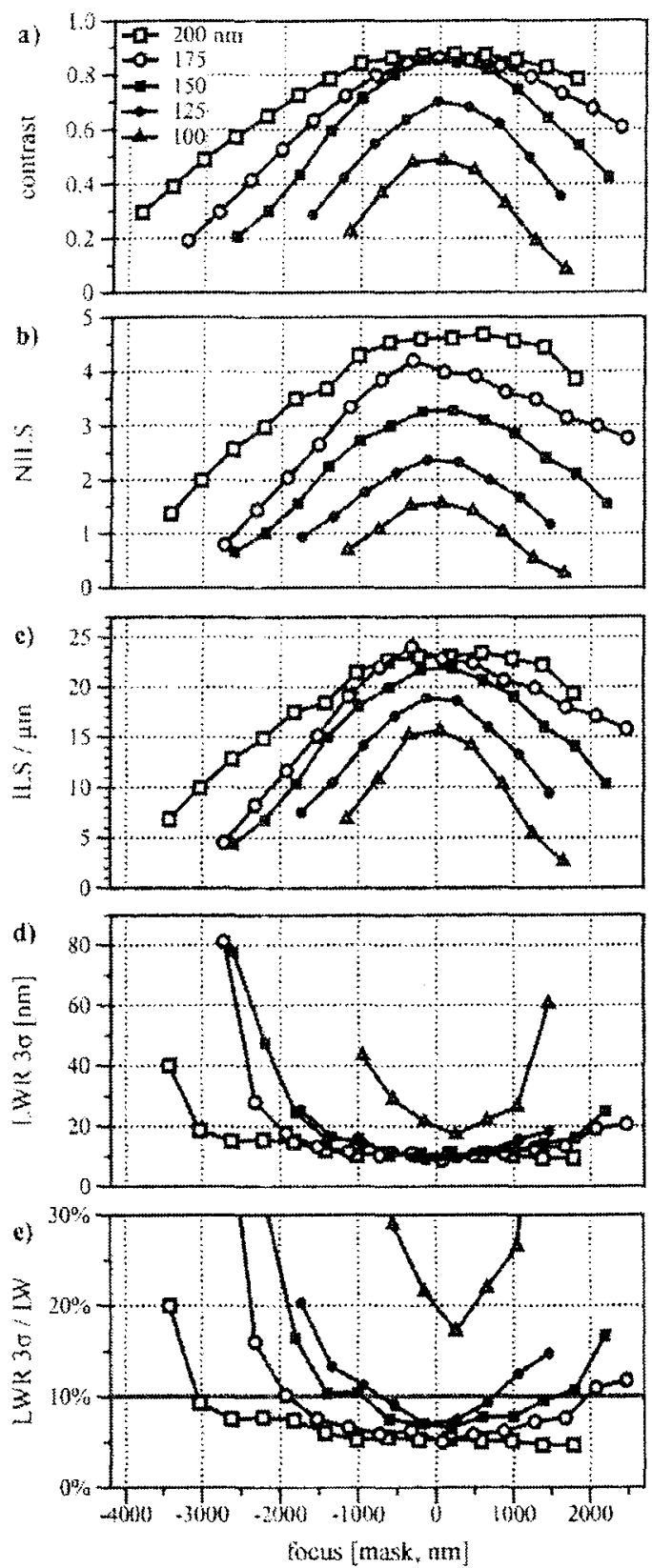

FlG. 6. Mask 2 line properties through-focus for half-pitch values between 100 and $200 \mathrm{~nm}$. (d) The flat lower limit of the LWR $3 \sigma$ value may arise from the inherent mask pattern roughness. (e) For half-pitch values of $125 \mathrm{~nm}$ and larger, LWR $3 \sigma$ values fall well below the $10 \%$ threshold commonly used to define defects, indicating that the AIT has the sensitivity to confidently detect defects for those line sizes.

variation of $4.0 \%$, rms. This contribution varies inversely with the NILS and therefore should not exhibit a flat lower limit when NILS increases. Using the peak NILS values for line sizes $\{100,125,150,175$, and $200 \mathrm{~nm}\}$ given in Table 1 , Eq. (6) predicts that this $4.0 \%$ intensity variation will contribute $(3.6,3.0,2.6,2.4$, and $2.4 \mathrm{~nm})$ or $\{2.8 \%, 1.9 \%, 1.4 \%$, and $1.1 \%\}$ to the relative linewidth variation, $\sigma_{\mathrm{LW}} / \mathrm{LW}$. This contribution is uncorrelated from other LWR sources and so adds in a root sum of squares manner. 


\begin{tabular}{|c|c|c|c|c|c|c|c|}
\hline Mask & $\begin{array}{c}\text { CD } \\
\text { (mask } \\
\text { (nm) }\end{array}$ & Contrast & $\begin{array}{c}1: 1 \\
\text { threshold }\end{array}$ & NIL.S & $\begin{array}{c}\text { ILS } \\
(\mu \mathrm{m})\end{array}$ & $\begin{array}{c}\text { LWR } 3 \sigma \\
\text { (mask) } \\
\text { (nm) }\end{array}$ & $\begin{array}{c}\text { LWR } 3 \sigma: \text { LW } \\
(\%)\end{array}$ \\
\hline 2 & 100 & 0.49 & 0.67 & 1.56 & 15.60 & 17.19 & 17.0 \\
\hline 2 & 125 & 0.70 & $0 . \overline{5} 5$ & 2.36 & 18.39 & 8.73 & 7.0 \\
\hline 2 & 150 & 0.86 & 0.46 & 3.28 & 21.88 & 9.94 & 6.6 \\
\hline 2 & 175 & 0.86 & 0.39 & 4.20 & 24.00 & 8.77 & 5.0 \\
\hline 2 & 200 & 0.88 & 0.34 & 4.68 & 23.00 & 9.36 & 4.7 \\
\hline l & 250 & 0.94 & 0.19 & 5.36 & 21.42 & 18.96 & 7.6 \\
\hline
\end{tabular}

Table I summarizes line properties at best focus for masks 1 and 2

\section{CONCLUSION}

We have used two masks to evaluate LWR and assess the pattern defect sensitivity of the AIT. These masks were typical of masks measured at the AIT on a routine basis. The AIT measures the reflected light intensity from a mask without the complications of photoresist. However, similar to photoresist exposures, the measured LWR is highly sensitive to systematic intensity variations and random noise sources. For many noise sources, including multilayer phase roughness, this sensitivity varies inversely with the NILS. So as NILS increases, the contributions from random sources to measured LWR will decrease.

Line properties such as contrast, image slope, and linewidth can be measured precisely by examining average intensity profiles from small detail regions. LWR measurements, however, rely on linewidth measurements from individual image rows, without averaging. Thus they are much more sensitive to the exposure level and are more vulnerable to noise sources. Our measurements with mask 1 showed that longer exposure times were needed to improve the LWR measurements.

For dense line patterns, the AIT zone plate microscope has demonstrated the sensitivity required to identify linewidth changes well below the $10 \%$ width-change threshold commonly used to classify defects.

In all line profile measurements, we observed a peak in the NILS value at best focus. Despite the NILS peak, however, LWR measurements from mask 2, for line sizes of $150 \mathrm{~nm}$ and larger, have a flat response near best focus that does not go below approximately $9 \mathrm{~nm}, 3 \sigma$. We attribute this behavior to the inherent pattern roughness on the mask itself. Independent pattern evaluations, through SEM measurements of the mask, have not yet been made. Yet this level is consistent with the LWR $3 \sigma$ values, observed by SEM, on other masks in our laboratory.

Further experiments are required to investigate the relationship between the exposure level and measured LWR.
Such studies could be used to set guidelines for accurate measurement with the AIT and other mask inspection microscopes that will be developed.

\section{ACKNOWLEDGMENTS}

The authors gratefully acknowledge the support of SEMATECH and many LBNL team members who make this research possible. The AIT's technical support team includes Nathan Smith, Charles D. Kemp, Paul Denham, Robert Gunion, Brian Hoef, Hanjing Han, Kenneth Woolfe, Jeffrey Gamsby, and Ron Tackaberry. The AIT's chief engineer is Senajith Rekawa. Contributing scientists include James Mac. dougall, Hakseung Han, and Bruno LaFontaine. This work is funded by SEMATECH under Project No. LITH-343S2 and was performed under the auspices of the U.S. Department of Energy by University of California Lawrence Berkeley National Laboratory under Contract No. DE-AC02$05 \mathrm{CH} 11231$.

K. A. Goldberg et al., Proc. SPIE 6730, 67305E (2007)

'K. A. Goldberg, I. Mochi, P. P. Naulleau, H.-S. Han. and S. Huh, Proc. SPIE 7122. 71222E (2008)

${ }^{3}$ K. A. Goldberg, I. Mochi, and S. Huh, Proc. SPIE 7271, 72713N (2009).

${ }^{4}$ I. Mochi, K. A. Goldberg, P. Naulleau, and S. Huh, Proc. SPIE 7271, $727123(2009)$.

${ }^{5}$ S. Huh, P. Kearney, S. Wurm, F. Goodwin, K. Goldberg, I. Mochi, and E. Gullikson. Proc. SPIE 7271, $72713 \mathrm{~J}$ (2009).

${ }^{6}$ S. Y. Lee et al. Proc. SPIE 7122, 71222I (2008).

${ }^{7}$ C. H. Clifford, S. Wiramadja, T. T. Chan. A. R. Neureuther, K. A. Goldberg, I. Mochi, and T. Liang, Proc. SPIE 7271. 727lIF (2009).

${ }^{3}$ C. H. Clifford, S. Wiratmadja, T. T. Chan, A. R. Neureuther, K. A. Goldberg. I. Mochi, and T. Liang (unpublished).

${ }^{9} \mathrm{~K}$. A. Goldberg et al., J. Vac. Sci. Technol. B 26, 2220 (2008).

10I. Mochi, K. A. Goldberg, R. Myakawa. P. Naulleau, and H. Han (unpublished).

"A. R. Neureuther and C. A. Mack, Optical Lithography Modeling, Handbook of Microlithography, Micromachining, and Microfabrication. edited by P. Rai-Choudhury (SPIE Press, Bellingham, Washington, 1997), Vol. 1. Chap. 7.

'M. Lowisch, U. Dinger, U. Mickan, and T. Heil, Proc. SPIE 5374. 53 (2004).

${ }^{13} \mathrm{C}$. A. Mack, Field Guide to Optical Lithography (SPIE Press, Bellingham, Washington, 2006)

${ }^{14} \mathrm{P}$. Naulleau, D. Niakoula, and G. Zhang, J. Vac. Sci. Technol. B 26. 1289 (2008).

${ }^{15} \mathrm{~J}$. W. Bossung, Proc. SPIE 100. $80(1977)$. 